\title{
NUEVAS CITAS DE ASCLEPIADACEAE PARA ARGENTINA
}

\author{
por SERGIO A. CACERES MORAL*
}

\section{Summary}

Two species not previously mentioned from Argentina are treated and illustrated: Oxypetalum molle Hook. et Arn. and Oxypetalum incanum Fourn. Oxypetalum appendiculatum Mart. et Zucc., Oxypetalum confusum Malme, Oxypetalum jörgensenii Meyer, Oxypetalum microphyllum Hook. et Arn., Oxypetalum pannosum Decaisne, Oxypetalum stipatum Malme, Funastrum flavum (Decaisne) Malme and Blepharodon lineare (Decaisne) Decaisne are recorded for the first time for the Corrientes flora.

\section{Introducción}

Al estudiar ejemplares de Asclepiadáceas coleccionados en Corrientes y Misiones, he podido constatar la presencia de Oxypetalum molle Hook. et Arn. y Oxypetalum incanum Fourn., que resultaron ser novedades para la flora argentina. Incluyo además en este trabajo ocho especies que no estaban registradas para la flora de la provincia de Corrientes.

De acuerdo al criterio de Meyer (1943:6), para la subdivisión del género Oxypetalum, ambas novedades para el país pertenecen al subgénero Oxypetalum por presentar caudículas ensanchadas con diente grande y notable.

* Instituto de Botánica del Nordeste (CONICET-UNNE), Casilla de Correo 209, 3400 , Corrientes, Argentina.

Agradezco a la Lic. Sara G. Tressens su asesoramiento en la elaboración de este trabajo y a la Srta. Lau ra Simón quien pasó a tinta las láminas. 


\section{Oxypetalum molle Hook. el Arn.}

Fig. 1

Hook. et Arn., J. Bot. 1:289. 1834. "St. Catherine, Tweedie 231". Fontella Pereira \& Marquete, N.F. da S, Bradea 1 (14): 130. 1972; Fontella Pereira,J, Tribuna Farm. $48(1-2): 99-100.1980$.

Oxypetalum nigrescens Fourn., in Martius, $\mathrm{Fl}$. bras. 6(4): 264.1885.

Oxypetalum suaveolens Fourn., in Martilis, Fl. bras. 6(4): 264.1885.

Gothofreda mollis (Hook. et Arn.) O. Kuntze, Revis. gen. pl. 2: 420. 1891.

Gothofreda suweolens (Foum.) 0. Ku ritze, Revis. gen. pl. 2: 420. 1891.

Gothofredc nigrescens (Fourn.) O. Kuntze, Revis. gen. pl. 2: 420. 1891.

Oxypetalum henschenii Malme, Kongl. Svenska Vetenskapsakad. Handl. 34(7):

45. 1900 .

Oxypetalum oliganthum Malme, Kongl. Svenska Vetenskapsakad. Handl. 34(7). 46. 1900.

Oxypetalum hirsutulum K. Schum., Bot. „ahrb. 30(67): 31.1901.

Oxypetalum campanulatum Handel-Mazzetti, Akad. Wiss. Wien., Math. -Naturwiss. Kl.,Denkschr. .9(2): 381. 1931.

Es una planta voluble que crece en bordes de selva. Presenta inflorescencias paucifloras, brevemente pedunculadas. Los lóbulos de la corola son verrucosos en la cara interna y presentan un leve reticulado castaño en la flor fresca. La corona es blanca, con lóbulos cuadranguiares provistos de un apéndice en la cara interna. El retináculo es laminiforme y algo convexo en vista lateraî. El apéndice estigmático es muy llamativo en la flor fresca pues tiene la base púrpura y el resto blanco.

En la clave de las especies argentinas (Veyer, 1943), la ubico próxima a Oxypetalum sublanatum Malme, cuya única cita para el país esta hecha sobre la base de un ejemplar de Molfino procedente de Misiones, San José de la Esquina, febrero 1922.

Fig. 1. Oxypetalum molle Hook. et Arn.: A, rama con flores; B, flor; C, lóbulo de la corona, cara extema, D y E, translator de perfil y de frente. (Vanni y Cáceres 644). 


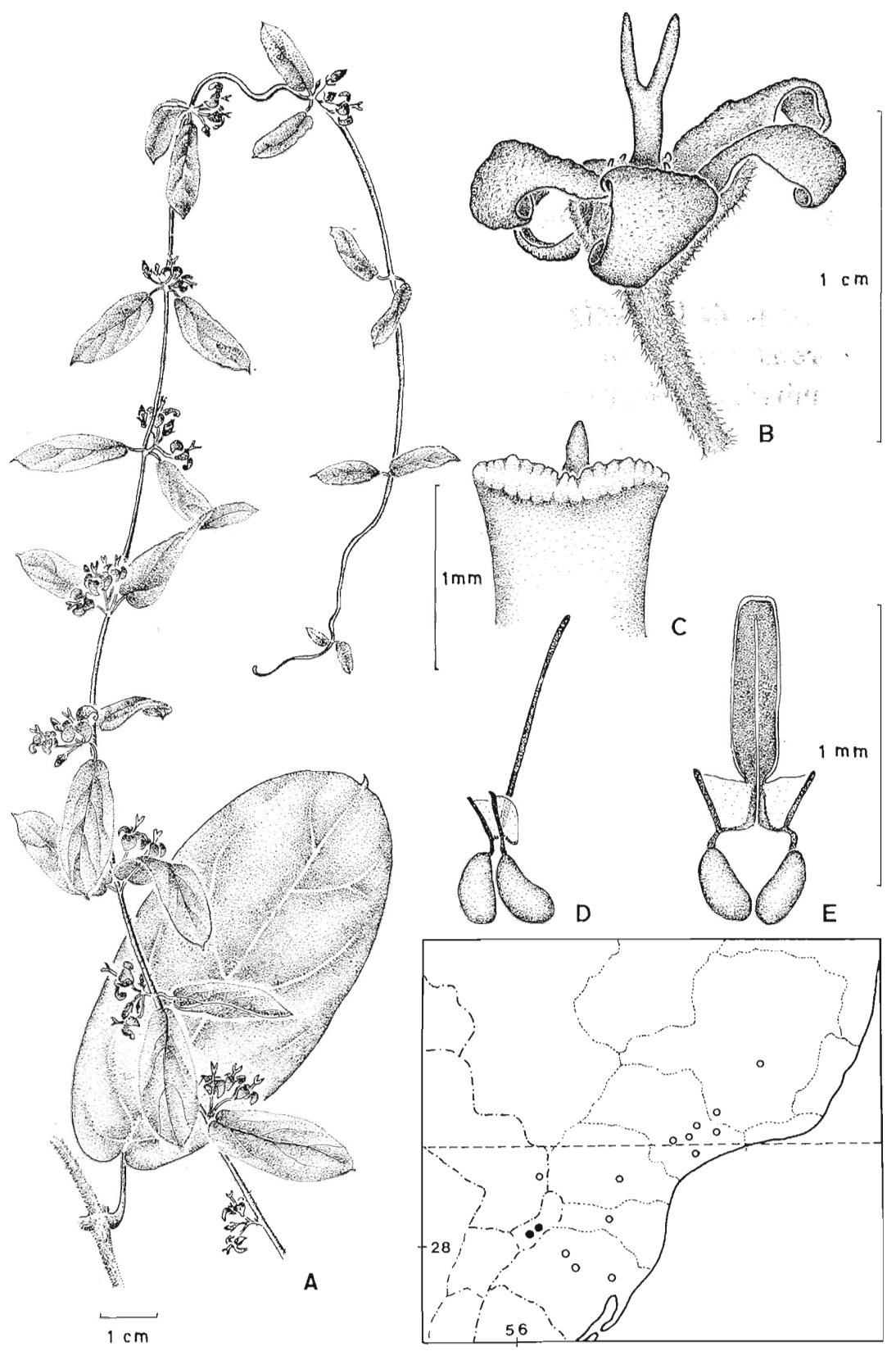


Ambas especies se pueden diferenciar por los siguientes caracteres:

A Lóbulos de la corola verrucosos en la cara interna; lóbulos de la corona con apéndice interno; apéndice estigmático bífido hasta la mitad; retináculo menor de $1 \mathrm{~mm}$.

O. molle

A' Lóbulos de la corola no verrucosos; lóbulos de la corona sin apéndice en la cara interna; apéndice estigmático grueso y cónico, levemente partido en el ápice; retináculo mayor de 1 $\mathrm{mm}$.

O. sublanatum

Oxypetalum incanum Fourn.

Fig. 2

Fournier, in Martius, Fl. bras. 6(4): 276. 1885. "Ad Corgo de.Jaragua: Pohl 1046". (Fototipo F 31862 , W!); "in prov. Goyaz: Riedel; in prov. S. Paulo: St. Hilaire".

Oxypetalum tridens Malme, Ark. Bot. 3(8): 8. 1904. (Fototipo F 26985, Hassler 9591, G!). Malme, Ark. Bot. 16(15): 27. 1920.

Es una planta erecta, vellosa, con indumento blanquecino. Hojas de hasta $7.5 \mathrm{~cm}$ long., con 2-4 glándulas caedizas en la base de la lámina. Las flores, vistosas de color castaño, se disponen en umbelas de hasta 7 flores que sobresalen entre las hojas. Las dimensiones del retináculo varían entre $0.83-0.96 \mathrm{~mm}$ long. en flores de una misma planta. Crece en campos entre matas de gramíneas y a veces junto a Oxypetalum confusum Malme, especie muy afín con la cual según Malme se hibridiza (1904: 13).

Otra especie muy próxima es Oxypetalum charrua Meyer, que crece en el $\mathrm{S}$ de Misiones. Se trata al parecer de una planta escasa, Meyer (1943: 45), menciona sólo el ejemplar tipo y Rambo (1958: 11.8), cita dos colecciones para Rio Grande do Sul, Brasil. 
Podemos diferenciar rstas especies afines con la siguiente clave:

A Pedicelos mayores de $10 \mathrm{~mm}$ long.; tubo corolino tan largo como la mitad de las lacinias del cáliz; corola castaño-purpúreo lóbulos erectos, carenados; lóbulos de la corona bífidos, con apéndice interno grande; retináculo más grande que los polinios con el ápice rurvado hacia afucra.

(). incanum

A Pedirelos menores de $10 \mathrm{~mm}$ long.; tubo corolino más a mienos de igual longitud que las lacinias del cáliz; corola de otro color. lóbulos suberectos, no sarenarlos; corona con lóbulos bitidos sin apéndice interno; retináculo $d e^{2}$ igual longitud que los polinios o más largo. pero con el ápice curvado hacia adentro.

B Retináculo lanceolado, aproximadamente de igual longitud que los polinios, ápice curvado hacia afuera: lóbulos de la corona con el ápice levemente doblado hacia adentro: apéndice esligmátice menor de $1 \mathrm{~mm}$ long.

(). (harma

B* Retináculo oblongo-alargado, de mayor longitud que los polnios, ápicr curvado hacia adentro: lóbulos de la rorona violáceos. ronniventes en ia parte superior, ápice no doblado hacia adentro: apéndicestigmático 1-- $1.5 \mathrm{~mm}$ long.

(). (1)n firsum

Para la flora de Correntes, además de () repelatum incammm Fourn., agrego tambien a Oxypetalum appendiculatum Mart. "l Zucc. citada para Brasl. Paraguay y Visiones, en Aroentina (Veyer 1943: 24); Oxvpelalum confusum Valme (Veycr 1943: t7) y ()x rpetalum jörensenn Vever (Mryer 194:3: 62: Rambo 19.58:

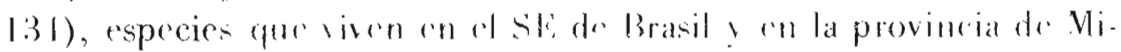


siones y que han sido halladas en campos del NE correntino; $O x y$ petalum microphyllum Hook. et Arn., mencionada para Brasil, Paraguay, Uruguay y las provincias de Misiones y Entre Ríos (Rambo 1958: 131; Meyer 1943: 41; Meyer y Bacigalupo 1979: 110); Oxypetalum pannosum Decaisne, crece en Brasil, Paraguay, Uruguay y en la provincia de Misiones (Lillo 1919: 413; Meyer 1943: 27; Meyer y Bacigalupo 1979: 115), fue coleccionada en bordes de selva muy próximas al límite con Misiones; Oxypetalum stipatum Malme, citada para Brasil, Paraguay y Misiones (Malme 1900: 44; Meyer 1943: 31), vive en selvas del NE de Corrientes.

Con estas nuevas citas se eleva a 30 el número de especies de este género que crecen en Argentina, siendo Misiones y Corrientes las provincias mejor representadas con 21 y 19 especies respectivamente.

Finalmente menciono por primera vez para Corrientes a Funastrum flavum (Decaisne) Malme, citada para Brasil, Uruguay y las provincias de Buenos Aires y Entre Ríos (Meyer y Bacigalupo 1979: 130), y a Blepharodon lineare (Decaisne) Decaisne, que habita en Brasil, Paraguay y Misiones (Lillo 1919: 433), siendo esta última la única especie del género que vive en el país.

\section{Material estudiado}

Blepharodon lineare: ARGENTINA, Prov. Corrientes, depto. Santo Tomé, ayo. Chimiray y ruta 40, 9-XII-1981, Tressens et al. 1858 (CTES, LIL, VEN, K).

Funastrum flavum: ARGENTINA, Prov. Corrientes, depto. Santo Tomé, Ea. Garruchos, potrero Puente, 11-II-1972, Krapovickas et al. 21 574. (CTES); Ea. Timbó, 28-II-1983, Schinini et al. 23599 (CTES, MBM. WIS), Ea. Vuelta del Ombú, $3 \mathrm{Km} \mathrm{SW} \mathrm{de} \mathrm{Gdor.} \mathrm{Vira-}$ soro, 10-XII-1984, Tressens et al. 2702 (CTES, GH, K, LIL, VEN); depto. Ituzaingó,

Fig. 2. Oxypetalum incanum Fourn., A, rama con llores; B, flor; C, lćbulo de la corona, vista lateral; D, ginostegio; $\mathrm{E}$, translator; vista lateral (Tressens et al. 2818). 


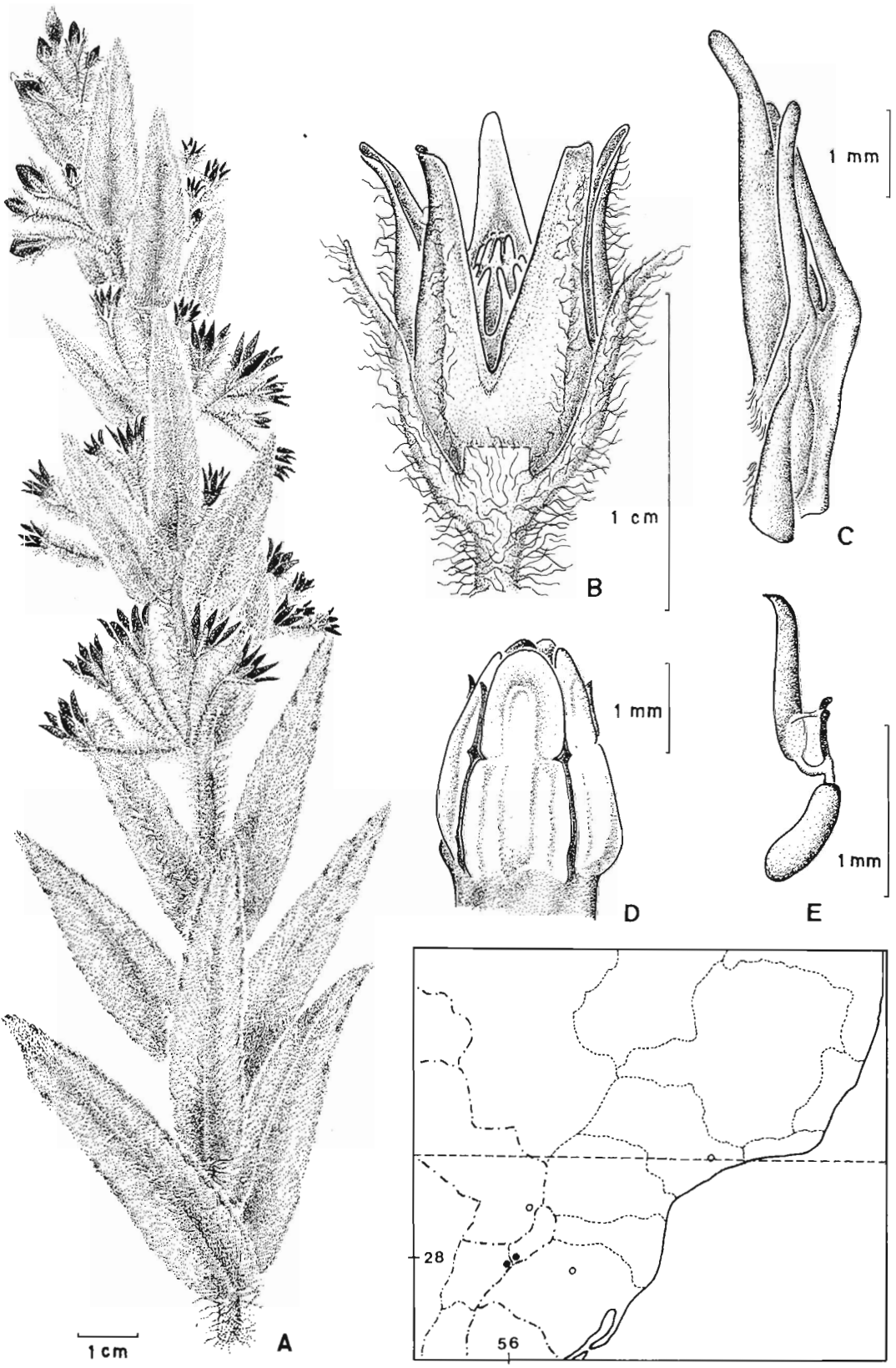


Mayadito. $20 \mathrm{~km} W$ de Apóstoles, 4-11-1982. Schinunı ei al 21792 (CTLS): $17 \mathrm{Kur}$ VA de San Carlos, Ea. Rincón Chico, 11-XII-1 1984, Tressens et al. 2710 (C.TLS)

Oxypetalum appendiculatum: ARGENTINA Prov Corrientes, depto. Santo lome. F. San Francisco, $23 \mathrm{Km} N \mathrm{~W}$ de Gdor Virasore. 2 XI: 1070, Krapovickas et al 16876 (CTES, LP, F. WIS, UC, BA, BAA, BAB, IAC, MO, LL): Garruchos rosta del rio liruguay, 12-IV_1974. Kraporickas et al 25033 (CTLS): Garruchos, Fa San Juan Bautista 20. IV .1974, Krapovickas et al. 25783 (CTES): Ayo. Chimiray, 23-IX-1974, Krapovic kas et al 26169 (CTES) Ea. Timbó, 1-III-1983, Schinini et al 23542 (C.THS, MICH) Ayo. Chimirav y rio liruguay, 13-XII-1984. Tressens et al 284.7 (CTFS): Ha (iarruchos. 18-1I 1960, TM Pedersen 5475 (Herb. T.M. Pedersen), depto ltuzaingó, Isla ipipi (irande, Panco Cué, 6-X-1978. Schinini y Vanni 15814 (CTES. SI, MO).

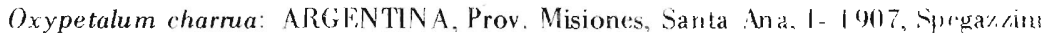
(LPS 13297, Tipo).

Oxypetalum confusum. ARGENTINA, Prov Corrientes, depto Santo Tomé. avo. Chi miray ! ruta 4.0, 9-XII-I 981. Tressens el al 1859 (CTES); Idem. 12-Xli-1984. Tressens it al 2816 (CTFs, C, UC): Ruta 40.6 $\mathrm{km} \mathrm{sW}$ de Colonia (rarabi (ayo. Ciriaco), 14 . X11 1984, Tressens et al 2875 (C.TES. I ( : ( ; , K); $33 \mathrm{Km} \vee$ de Santo Tome. ruta pror 10. 27-I-1976. Krapourkas y (.ristóbal 28962 (CTES. LIL): $21 \mathrm{Km} N$ de Santo T'omé. ruta 40, 14-XI-1980, O thumada v S; hınini 4075 (CTES) depto. I tuzaingó, $12 \mathrm{~km}$ Vi de ruta nac. 12. camino a San (arlom. 10-11--1971, Krapovickas et al 17896 (CWFS): rula 39. aprox $27 \mathrm{Km}$ Vt de ruta 14.11 YII-1981, Carnevali 4970 (CTES).

Oxypetalum incanum ARGENTINA, Prov. Corrientes, depto Santo Tome ayo. Chi mikay y ru ta 40,9-XII I08J. Tressens et al 1860 (CTIS, RB.VEN):Idem. I2-XII-I 984. Tressens et al. 2818 (CTES, LIL, GH, K) l'rov Misiones, depto Apósloles. Tres Galpones* 8-II-1947. lluidobro t810(LLL).

Oxypetalum lörgenst:aii: ARGENTINA, Prov Corrientes. depto. Santo Tomé, Ea. Garru (hos, carhuera, a o. Chimiray, $6-11-1972$, Krapovickas el al 21145 (CTES) Ea limbó. polreroluna. $28^{\circ} 21^{\prime}$ 's y $55^{\circ} 47$ it 8-XII-1981. Tresens etal. 1661 (CTLS).

Oxverlalum mecrophyllum ARGENTINA. Prov Corrienles, deplo Itukaingo, I.j Km fideruta nae 12, camino a San Carlos, $11-13-11-1971$. Krapovirkas etal. 18016 (CTLS): F.a. Santa Kita. $56^{\circ} 4^{\prime} \mathrm{W}, 27^{\circ} 3^{\circ} \mathrm{S}$. avo proximo al caseo. 4-111-1987. Krapovickas et at

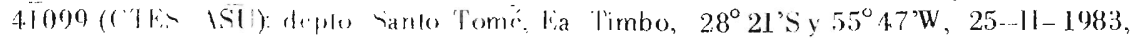

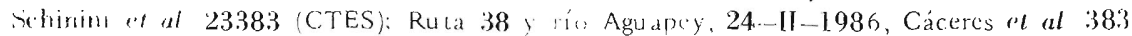
(CTES): depte Vereder. nula de Vercedes a Paso de los libres. $45 \mathrm{Km}$ St de Vereedes,

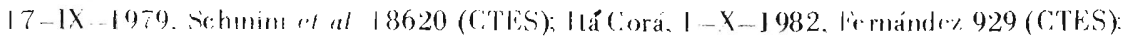
$11 \mathrm{~km}: \mathrm{se}$ Mricedes antiguo camino a Cu ruzí Cuatiá, Co. Pajaribo, 23-11-1984. Tressens

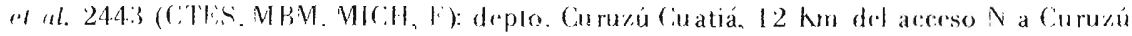

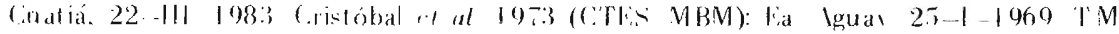

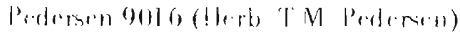

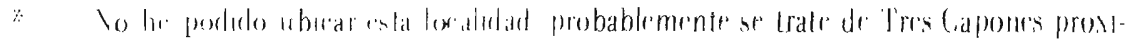

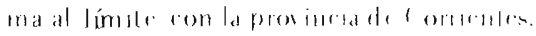


Oxypetalum molle: ARGENTINA, Prov. Misiones, depto. Cainguás, alrededores d: Campo Grande, 25-XI-1986, Vanni y Cáceres 64.4 (CTES, K, GH, LIL. VEV); Idem 26 -XI-1986, Vanni y Cáceres 684 (CTES); depto. Oberá, $3 \mathrm{Km} S$ de Guaraní. 26 XII . 1973, Pire y Mroginski 20! (CTES, G, RB, MBM). BRASIL, Est. Paraná, Mun. Pitanga. Barboleta, 13_XII_1973, Hatschbach 33513 (CTES); Mur. Londrina, Serra do Arreio, 17--XI-1969, Hatsehbach 22917 (CTES). PARAGUAY, depto. Nlo Paraná, 1909/10. Fiebrig 6021 (LII).

Oxyprtalum pannosum ARGENTINA. Prov. Corrientes, depto Santo Tomé, Fa. (,arruchos. 8-11-1972, Krapovickas et ai 21392 (C.TES, MBN, G, CONC) Idem. 18II 1960. T.M Pedersen 54.76 (Herb. T.M Pedersen); Ayo. Chimiray y ruta 40, 9-X]11981, Tressens el al 1861 (C'TES. LIL): Idem. 28-XI-1 985, Cristóbal et al. 2045 (CTES).

Oxypelalum slipatum ARGENTINA, Prov. Corrientes, depto. Ituzaingó, ruta 39. $11.5 \mathrm{~km} S \mathrm{de}$ ruta 12.9 XII-1987, Vanni y Radovancich 1021 (CTFS), depto. Santo Tome, fia. Vuelta del (mbu, $3 \mathrm{Km} \mathrm{SW}$ de Gdor Virasoro. 10-XJI-1984, Tressens et al. 2679 (C.TLS. LII, VF, V. RB, K, GH, F. (G).

Oxypetalum sublanatum. BRASli, Est Parana, Mun. Ortigueira, Serra dos Mulatos, 18 XI -1969, Hatschbach 22928 (CTES); Mun. Pirai do Sul, Joaquim Murtinho, 18-XI1976, Hatschbach 39191 (CTES); Serra de São Luis, BR 277, 19-I-1985, lie rrucci et al. 233 (CTLS): list. Santa Catarina, Parque das Pedras Brancas, $10 \mathrm{Km}$ SE de Lages, 19-I 1988. Krapor ickas y Cristóbal 42103 (CTES).

\section{Bibliografía}

Hoehne. F.C. 1916. Monographia das Asclepiadaceas Brasileiras. Oxypelalum R. Br. Comm. Linh. Telegr. Fistrat. Matto-..Grosso ao Amazonas 38(1): 1-131.

fiontella Pereira, J. \& Marquete, N.F. da S. 1972. Estudos em 1sckepuadacear. I. Novos sinónimos. Bradea I (14): 129-136.

liontella Perrira, J. 1980. Contribuscão do estudo das 1srlepadaréae Brasileiras. YV. Tribuna Farm. $48(1-2): 93-113$.

Lillo, V. 1919. Las Asclcpiadáceas argentinas. Physis 4: 410_137. Nalme. (i.O.A. 1904. Oxypetali species novae vel ab aurtoribus saepr confusate. Ark. Bot. 3(8): $1-19$.

1908. Fisperes et unités nouvelles de la flore paraguayenne. Bull. Herb. Boissier 8(6): 39.5 40)1. 
Malme, G.O.A. 1920. Asclepiadaceae Riograndenses adjectis notulis de ceteris Asclepiadaceis in Brasilia extratropica, Uruguay et Misiones collectis. Ark. Bot. 16(15): 1--34.

Matos-Araujo, P.A. de 1950. Contribuição ao conhocimento da familia Asclepiadaceae, no Brasil. Rodriguesia 13(25): 5-224. Meyer, T. 1943. Revisión de las especies argentinas del género Oxypetalum, Asclepiadaceae. Lilloa 9: 5-72.

1944. Asclepiadaceae, en Descole, Genera et Species Plantarum Argentinarum 2: 1-274.

Meyer, T. y Bacigalupo, N. 1979. Asclepiadaceae, en Burkart, Flora Mustrada de Entre Ríos (Argentina) 5: 103-147.

Occhioni, P. 1956. Contribuição ao estudo do gênero Oxypetalum. Arch. Jard. Bot. Rio de Janeiro 14: 37-210.

Rambo, B. 1958. Die Gattung Oxypetalum in Rio Grande do Sul, Südbrasilien. Sellowia 9: 117-145. 